\title{
Measurement of neutron scattering from noble gas to search for a short-range unknown force
}

\author{
Noriko OI $^{* A}$, Hirohiko M. Shimizu ${ }^{A}$, Katsuya Hirota ${ }^{A}$, Masaaki Kitaguchi $^{A}$, \\ Christopher C. Haddock ${ }^{B}$, William M. Snow ${ }^{B}$, Tamaki Yoshioka ${ }^{C}$, Satoru \\ Matsumoto $^{C}$, Kenji Mishima ${ }^{D}$, Takashi Ino ${ }^{D}$, Tatsushi Shima ${ }^{E}$ \\ ${ }^{A}$ Department of Physics, Nagoya University \\ Furo-chou, Chikusa-ku, Nagoya-shi, Aichi, Japan 464-8602 \\ ${ }^{B}$ Department of Physics, Indiana University \\ 727 E. Third St., Swain Hall West, Room 117, Bloomington, IN 47405-7105 \\ ${ }^{C}$ Department of Physics, Kyushu University \\ 744, Motooka, Nishi-ku, Fukuoka, Japan 819-0395 \\ ${ }^{D}$ High Energy Accelerator Research Organization, KEK \\ 1-1 Oho, Tsukuba, Ibaraki, Japan, 305-0801 \\ ${ }^{E}$ Research Center for Nuclear Physics, Osaka University \\ 10-1 Mihogaoka, Ibaraki, Osaka, 567-0047
}

E-mail: norikodphi.phys.nagoya-u.ac.jp

\begin{abstract}
We are searching for an unknown force that could couple to mass using neutron scattering from a noble gas. The neutron is a chargeless massive particle with a long lifetime, which consequently is suitable for the precision measurement of a small interaction with a range of the order of $1 \mathrm{~nm}$ by measurements of the momentum transfer distribution. We measured neutron scattering at the low-divergence beam branch on the BL05 NOP beamline in the Materials and Life Science Experimental Facility (MLF) at the Japan Proton Accelerator Research Complex (J-PARC). We measured $10^{5}$ scattering events and report our most recent analysis.
\end{abstract}

The 26th International Nuclear Physics Conference

11-16 September, 2016

Adelaide, Australia

* Speaker. 


\section{Introduction}

Several models that extend the Standard Model of Particle Physics have been discussed[U, ■, [3]. Certain models predict the existence of non-Newtonian gravity or a new possible force that couples to mass whose effects can be seen at short-range. When two masses $\left(m_{1}\right.$ and $\left.m_{2}\right)$ are separated by distance $r$, an unknown interaction potential $V(r)$ that couples to the masses is expressed as Yukawa-type potential:

$$
V(r)=-\frac{G_{N} m_{1} m_{2} \alpha}{r} \exp \left(-\frac{r}{\lambda}\right)
$$

where $G_{\mathrm{N}}$ is the gravitational constant, $\lambda$ is a Compton wave length of new boson and $\alpha$ is a coupling constant. An experimental constraint map is shown Figure $\mathbb{W}$. Constraint curves above $\sim 10 \mathrm{~nm}$ were deduced from macroscopic tests using torsion balances or isoelectronic techniques [四, [1, 目]. Constraint curves for smaller length scales were deduced from microscopic tests using neutrons or atoms $[\square,[]$. We are most sensitive to interactions below $10 \mathrm{~nm}$.

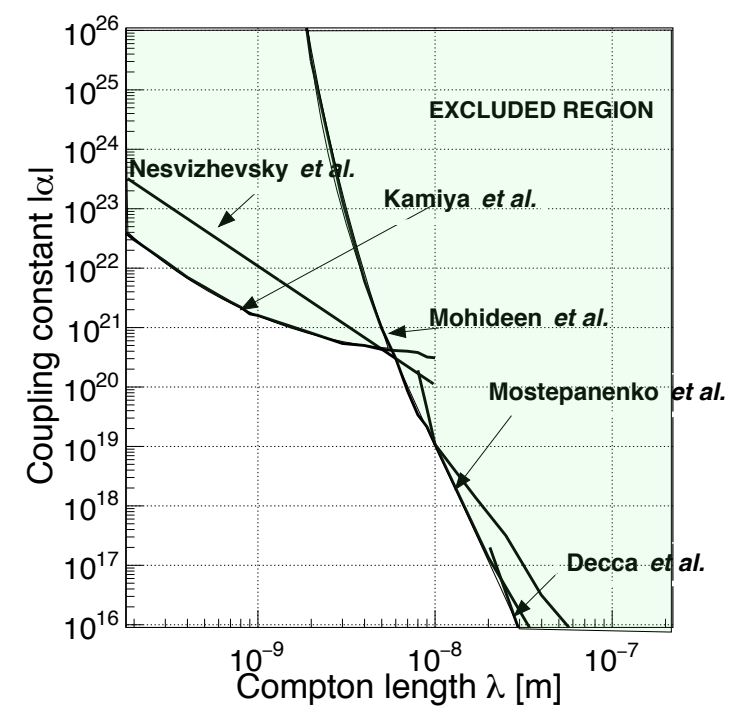

Figure 1: The experimental constraint map for $\lambda$ (Compton wavelength) and $\alpha$ (coupling constant). Green area is the excluded region ( $95 \%$ confidence level).

\section{Experimental principle}

We measure neutron scattering from a noble gas to search for unknown interactions. There are two good reasons to use neutrons:

(a) Neutron is neutral.

Electromagnetic interaction and Van der Waals force are suppressed.

(b) Neutron is massive.

Neutron is sensitive to forces that couple to mass. 
Additionally, there are three good reasons to use a noble gas as a target:

(a) Noble gas has no molecular or crystal structure.

When the scattering target contains structure, neutron scattering may show Bragg diffraction.

(b) Noble gas has zero atomic spin.

We do not need to consider multipole effects.

(c) Noble gas has chemical stability.

Experimental conditions do not change when the experiment is running.

If a Yukawa-type unknown interaction exists, neutron scattering will have an angular distribution. The additional scattering amplitude given in Eq. (ㅍ. $\mathbb{d}$ can be expressed as

$$
f_{Y}(\boldsymbol{q})=\frac{2 \alpha G_{\mathrm{N}} m_{\mathrm{n}}^{2} M}{\hbar^{2}} \frac{1}{\frac{1}{\lambda^{2}}+q^{2}}
$$

where $m_{\mathrm{n}}$ is neutron mass, $M$ is scattering target mass and $q$ is momentum transfer. Therefore, the differential scattering cross section including a Yukawa-type interaction is written as

$$
\begin{aligned}
\frac{\mathrm{d} \sigma}{\mathrm{d} \Omega} & =\left(b_{\mathrm{c}}+b_{\mathrm{e}}\left(1-Z f_{\mathrm{e}}(\boldsymbol{q})\right)+f_{\mathrm{Y}}(\boldsymbol{q})\right)^{2}+b_{\mathrm{i}}^{2} \\
& \simeq b_{\mathrm{c}}^{2}+2 b_{\mathrm{c}} f_{\mathrm{Y}}(\boldsymbol{q})+b_{\mathrm{i}}^{2}
\end{aligned}
$$

where $b_{\mathrm{c}}(4.92 \mathrm{fm}$ for Xe $[\mathrm{Q}])$ is coherent scattering length, $b_{\mathrm{i}}(3.04 \mathrm{fm}$ for Xe [Q] $)$ is incoherent scattering length, $b_{\mathrm{e}}$ is neutron-electron scattering length $\left(1.32 \times 10^{-3} \mathrm{fm}\right.$ for Xe [एU]]), $Z$ is target charge and $1-Z f_{\mathrm{e}}(\boldsymbol{q})$ is the electric form factor for the target atoms. The neutron-electron scattering is backward scattering and negligibly small in our experiment. Nuclear scattering distribution is isotropic at the nm-scale so we can search for the unknown force by precise measurement of the neutron scattering distribution (Figure 『). 


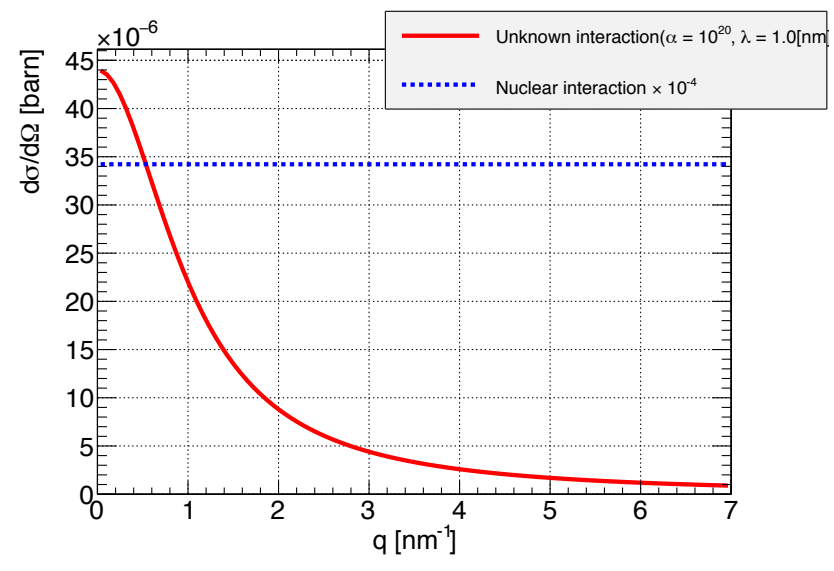

Figure 2: The differential scattering cross section of nuclear scattering (Blue dashed line) and that of Yukawa-type interaction (Red curve) when the scattering target was Xe gas. The parameter of $\lambda$ and $\alpha$ were assumed $1.0 \mathrm{~nm}$ and $10^{20}$, respectively. Nuclear scattering was scaled to $10^{-4}$.

\section{Facility and Setup}

Our experiment was performed at the low-divergence beam branch on the BL05 NOP beamline in the Materials and Life Science Experimental Facility (MLF) at the Japan Proton Accelerator Research Complex (J-PARC)[W]. We used a pulsed neutron beam in order to use the method of time of flight and determine the momentum transfer over a wide range with minimized waste of neutrons. The neutron beam intensity is about $2.7 \times 10^{6} \mathrm{n} / \mathrm{sec} / \mathrm{MW}$ and the neutron wave length we used ranged from $0.22 \mathrm{~nm}$ to $0.89 \mathrm{~nm}$. A slit and a collimator were placed $12 \mathrm{~m}$ and $16 \mathrm{~m}$ downstream of the neutron moderator, respectively. The size of the slit was $6 \mathrm{~mm}$ (horizontal) $\times 44 \mathrm{~mm}$ (vertical) and the diameter of the collimator was $10 \mathrm{~mm}$. Therefore, the neutron beam divergence was $1.50 \mathrm{mrad}$ (horizontal) and $11.5 \mathrm{mrad}(\mathrm{vertical})$. A schematic drawing of the experimental setup is shown Figure [3].

The gas cell has an inner volume of diameter $50 \mathrm{~mm}$ and length $145 \mathrm{~mm}$. Windows of the gas cell are made of $0.1 \mathrm{~mm}$-thick aluminum. The maximum pressure the gas cell can handle is $100 \mathrm{kPa}$. To suppress neutron scattering due to outgassing such as $\mathrm{H}_{2} \mathrm{O}$ or hydrocarbons, molecular sieves were put in the gas cell.

Neutrons were detected using a ${ }^{3} \mathrm{He}$ position sensitive detector (HePSD). The HePSD is composed of 7 proportional counter $1 / 2$ inch tubes filled with ${ }^{3} \mathrm{He}$ at a pressure of $10 \mathrm{~atm}$. Applied voltage for the proportional counter was $1530 \mathrm{~V}$. The size of the HePSD is $600 \mathrm{~mm} \times 90 \mathrm{~mm}$ and the detection efficiency was calculated as $93.9 \%$ for a neutron of wave length $0.3 \mathrm{~nm}$. 


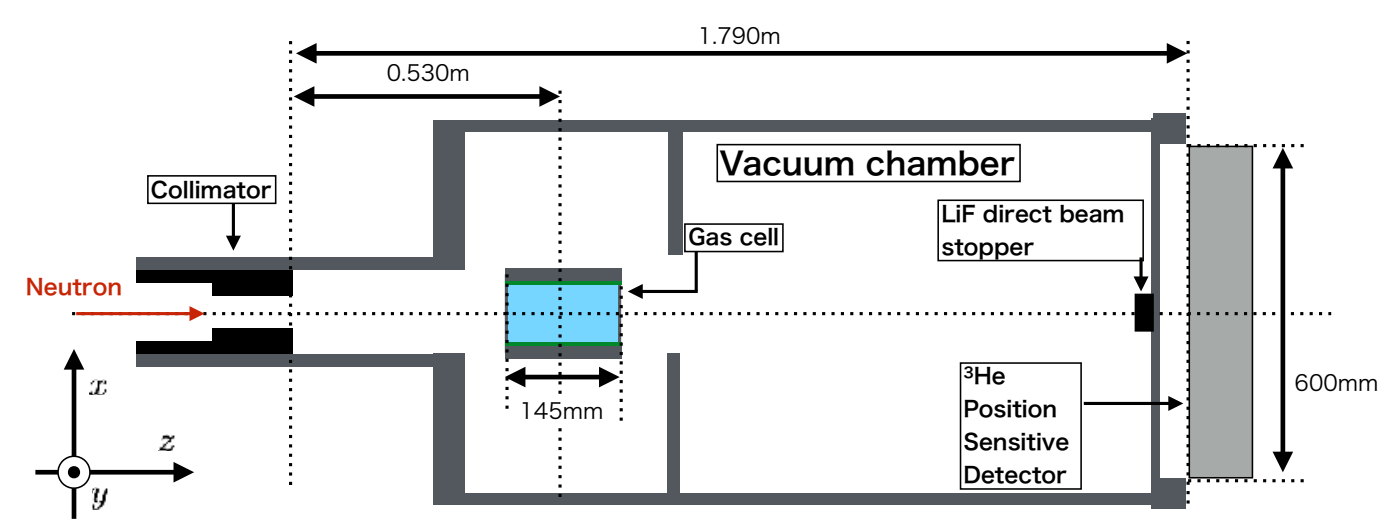

Figure 3: The schematic experimental setup from $16 \mathrm{~m}$ downstream of the neutron moderator to the detector(not to scale).

\section{Data analysis}

We used Xe gas as the scattering target. The backgrounds in our experiment are

(a) room background.

(b) neutrons scattered by the gas cell windows.

(c) neutrons scattered by the vacuum chamber.

We then took data using

(a) the empty cell.

(b) the cell filled He gas with the same pressure as Xe gas.

The data of Xe cell was then subtracted by the data of empty cell to estimate neutron-Xe scattering (Figure 耳).

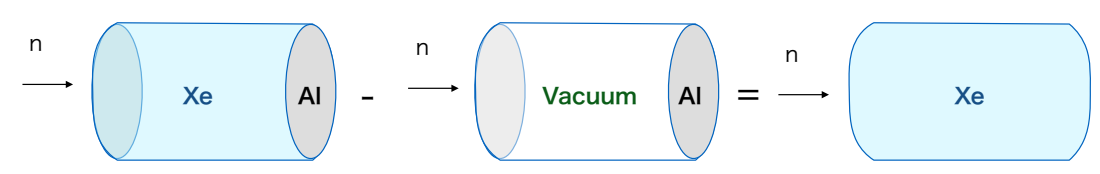

Figure 4: Neutrons scattered by the gas cell windows are included in the Xe cell data. We then subtract the empty cell data from the Xe cell data.

In addition we developed a Monte-Carlo simulation to estimate the scattering distribution more precisely. In the simulation, the effects of gas motion and absorption are included but the effects of 
multiple scattering were not included. The effect of the gas motion was estimated by the dynamical structure factor $S(\boldsymbol{q}, \omega)$ where $\omega$ is the energy transfer. $S(\boldsymbol{q}, \omega)$ is written as

$$
S(\boldsymbol{q}, \omega)=\left(\frac{\beta M}{2 \pi \hbar^{2} q^{2}}\right)^{1 / 2} \exp \left[-\frac{\beta M}{2 \hbar^{2} q^{2}}\left(\hbar \omega-\frac{\hbar^{2} q^{2}}{2 M}\right)^{2}\right]
$$

where $\beta=1 / k_{\mathrm{B}} T$ and $T$ is gas temperature [ㅁ] ]. Figure $\square$ shows the comparison between the experimental data and the simulation result.

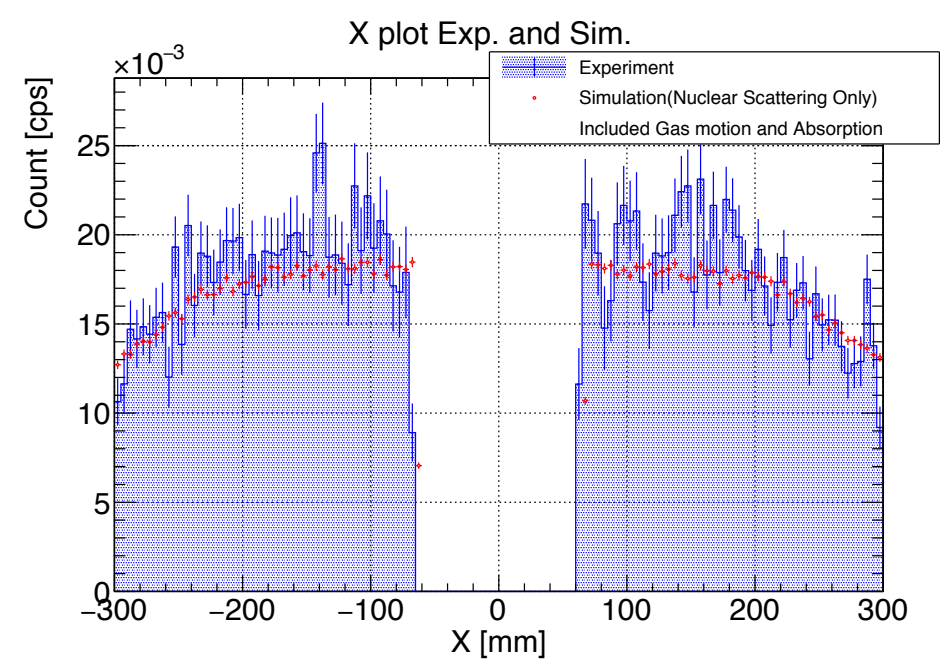

Figure 5: Comparison between the experimental data and the simulation result. $\mathrm{X}$ is the detector horizontal position. In the simulation the effects of the gas motion and the absorption were included but the effects of multiple scattering were not included. 
Preliminary plots of the differential scattering cross section from the experimental data are shown Figure 6. Time of flight is proportional to neutron velocity $v$. Also, the neutron wave number $\boldsymbol{k}$ is expressed as

$$
\begin{aligned}
|\boldsymbol{k}| & =\frac{m_{\mathrm{n}}|\boldsymbol{v}|}{\hbar} \\
& =\frac{m_{\mathrm{n}} L}{\hbar t}
\end{aligned}
$$

where $L$ is the flight path of neutrons and $t$ is time of flight. The momentum transfer $\boldsymbol{q}$ is written as

$$
q^{2}=4 k^{2} \sin ^{2}\left(\frac{\theta}{2}\right)
$$

where $\theta$ is the scattering angle. We can see and separate the background that depends on wave length and scattering angle as shown in the left of Figure 6 because we used a pulsed neutron beam. We estimated a coupling strength for the unknown interaction to be $\alpha \simeq 10^{22}$ where $\lambda=1 \mathrm{~nm}$ by this measurement. Therefore, with a factor of of $100 \sim 400$ increase in statistics we predict that we will be competitive with recent limits on $\alpha$ below $10 \mathrm{~nm}$.

Xe-Vac Diff. Cross Section

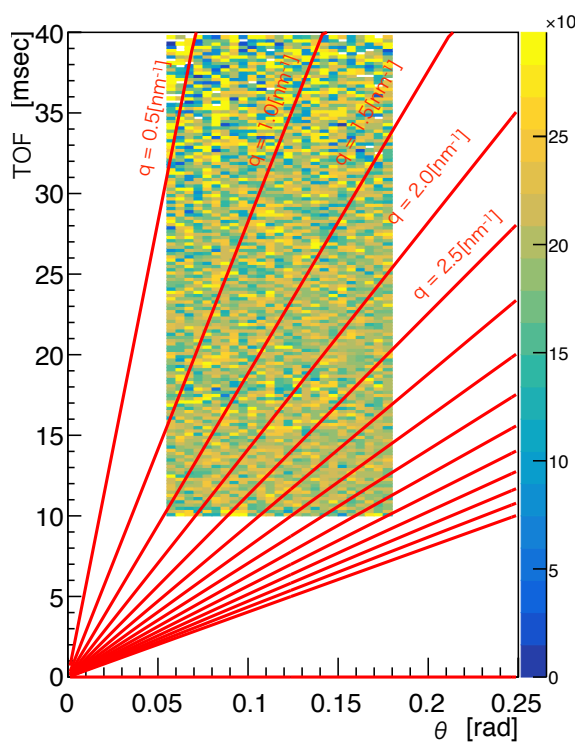

$\mathrm{d} \sigma / \mathrm{d} \Omega \mathrm{Xe}$

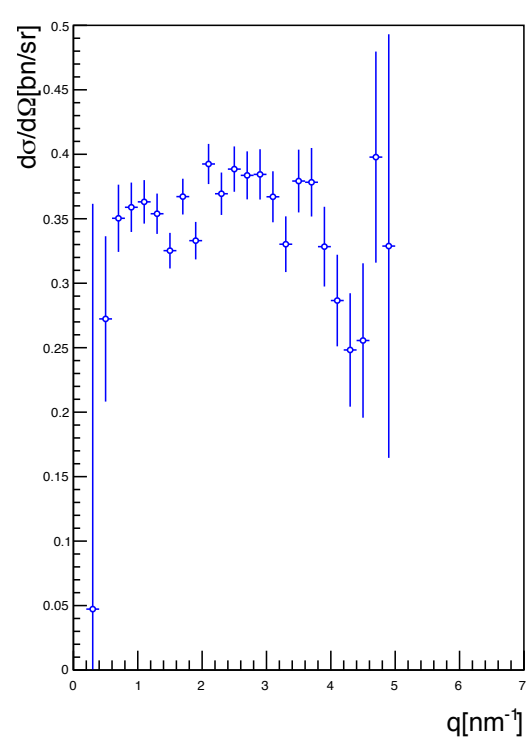

Figure 6: The preliminary plots of the differential scattering cross section from the experimental data. In the left figure, $\mathrm{X}$ axis is the scattering angle, $\mathrm{Y}$ axis is Time of Flight, $\mathrm{Z}$ axis is the differential scattering cross section and red line is the momentum transfer $q=2 k \sin \theta / 2$. Right figure is drawn using the left plot, where the $\mathrm{X}$ axis is the momentum transfer and $\mathrm{Y}$ axis is the differential scattering cross section. 


\section{Summary and future plan}

We measured the neutron scattering distribution to search for an unknown interaction at the low-divergence beam branch on the BL05 NOP beamline in the Materials and Life Science Experimental Facility at the Japan Proton Accelerator Research Complex. We reached a coupling strength for the unknown interaction to be $\alpha \simeq 10^{22}$ where $\lambda=1 \mathrm{~nm}$ by our recent measurement. We will improve our statistics by increasing the Xe gas pressure in the gas cell and using a larger beam size. In addition, we will upgrade our simulation and estimate the neutron scattering distribution more precisely.

\section{References}

[1] Nima Arkani-Hamed, Savas Dimopoulos, and Gia Dvali. The hierarchy problem and new dimensions at a millimeter. Physics Letters B, Vol. 429, No. 3-4, pp. 263-272, 1998.

[2] Nima Arkani-Hamed, Savas Dimopoulos, and Gia Dvali. Phenomenology, astrophysics, and cosmology of theories with submillimeter dimensions and $\mathrm{TeV}$ scale quantum gravity. Physical Review D, Vol. 59, No. 086004, 1999.

[3] YASUNORI FUJII. Yasunori fujii. nature physical science, Vol. 234, pp. 5-7, 1971.

[4] E. Fischbach, D. E. Krause, V. M. Mostepanenko, and M. Novello. New constraints on ultrashort-ranged yukawa interactions from atomic force microscopy. Physical Review D, Vol. 64, p. 075010, 2001.

[5] M. Bordag, U. Mohideen, and V.M. Mostepanenko. New developments in the casimir effect. Physics Reports, Vol. 353, No. 1-3, pp. 1-205, 2001.

[6] R. S. Decca, D. López, H. B. Chan, E. Fischbach, D. E. Krause, and C. R. Jamell. Constraining new forces in the casimir regime using the isoelectronic technique. Physics Review Letters, Vol. 94, p. 240401, 2005.

[7] V. V. Nesvizhevsky, G. Pignol, and K. V. Protasov. Neutron scattering and extra-short-range interactions. Physical Review D, Vol. 77, p. 034020, 2008.

[8] Y. Kamiya, K. Itagaki, M. Tani, N. Kim, and S. Komamiya. Constraints on new gravitylike forces in the nanometer range. Physics Review Letters, Vol. 114, p. 161101, 2015.

[9] Neutron scattering lengths and cross sections. https://www.ncnr.nist.gov/resources/n-lengths/.

[10] V. E. Krohn and G. R. Ringo. Measurement of the electron-neutron interaction by the asymmetrical scattering of thermal neutrons by noble gases. Phys. Rev., Vol. 148, pp. 1303-1311, 1966.

[11] Kenji Mishima, Takashi Ino, Kenji Sakai, Takenao Shinohara, Katsuya Hirota, Kazuaki Ikeda, Hiromi Sato, Yoshie Otake, Hitoshi Ohmori, Suguru Muto, Norio Higashi, Takahiro Morishima, Masaaki Kitaguch, Masahiro Hino, Haruhiko Funahashi, Tatsushi Shima, Jun ichi Suzuki, Koji Niita, Kaoru Taketani, Yoshichika Seki, and Hirohiko M. Shimizu. Design of neutron beamline for fundamental physics at j-parc BL05. Nuclear Instruments and Methods in Physics Research Section A:

Accelerators, Spectrometers, Detectors and Associated Equipment, Vol. 600, No. 1, pp. 342 - 345, 2009.

[12] Stephen W. Lovesey. Theory of Neutron Scattering from Condensed Matter, Vol. 1. Clarendon Press, 1984. 